\title{
A Generalisation of the Cauchy-Kovalevskaïa Theorem
}

\author{
Mauricio Garay ${ }^{1}$
}

Received: 1 July 2015 / Revised: 15 May 2016 / Accepted: 23 June 2016 / Published online: 9 August 2016 (C) Institute for Mathematical Sciences (IMS), Stony Brook University, NY 2016

\begin{abstract}
We prove that time evolution of a linear analytic initial value problem leads to sectorial holomorphic solutions in time.
\end{abstract}

Keywords Cauchy-Kovalevskaïa theorems · Initial value problem · Characteristic Cauchy data $\cdot$ Heat equation

Mathematics Subject Classification 35A10

\section{Introduction}

Among the class of systems of partial differential equations, evolutionary ones form a minority. They are nevertheless of considerable importance because they describe time evolution of physical data. This can already be seen for ordinary differential equations where, among differential equations, vector fields deserve a particular attention. The aim of this paper is to prove that evolutionary linear partial differential systems always admit sectorial analytic solutions, where the width of the sector depends on the regularity of the initial condition.

Before stating our main theorem, let us recall the results obtained by Kovalevskaïa in her thesis (von Kowalevsky 1875) (see also Audin 2008 for historical aspects).

We consider the vector space $\mathbb{C}^{n}$ with coordinates $z_{1}, \ldots, z_{n}$ and let $\mathcal{O}_{n}$ be the algebra of germs of holomorphic functions at $0 \in \mathbb{C}^{n}$ (series in $z_{1}, \ldots, z_{n}$ which are analytic in some neighbourhood of the origin). We put

Mauricio Garay

garay91@gmail.com

1 Lycée Franco-Allemand, Rue Collin Mamet, 78530 Buc, France 


$$
\partial^{I}:=\partial_{z_{1}}^{i_{1}} \partial_{z_{2}}^{i_{2}} \ldots \partial_{z_{n}}^{i_{n}}, \quad I=\left(i_{1}, \ldots, i_{n}\right)
$$

and define the order $\sigma(I)$ of the operator $\partial^{I}$ as the sum of the coordinates of the vector $I \in \mathbb{N}^{n}$ :

$$
\sigma(I):=i_{1}+\cdots+i_{n}
$$

An evolution equation of order $s$ is a system of partial differential equations of the form

$$
\partial_{t} u=F\left(u, \partial^{I_{1}} u, \ldots, \partial^{I_{k}} u\right), \quad \sigma\left(I_{j}\right) \leq s, u=\left(u_{1}, \ldots, u_{m}\right), z=\left(z_{1}, \ldots, z_{n}\right)
$$

with some initial condition $u(t=0, \cdot)=u_{0}$, where $F$ is a holomorphic map.

Kovalevskaiia proved that the formal solution to such an initial value problem:

$$
u(t, \cdot):=\sum_{k \geq 0} u_{k} t^{k}, \quad u_{k} \in \mathcal{O}_{n}^{m}
$$

exists and is unique. Then she proceeded to the analytic properties of time evolution. For $s=1$, she showed that the formal solution is holomorphic, in any sufficient small neighbourhood of the origin in $\mathbb{C}^{n+1}$. A result now called the Cauchy-Kovalevskaïa theorem. For $s=2$, Kovalevskaïa considered the particular case of the one dimensional heat equation and discovered that the formal solution might be divergent.

To state Kovalevskaïa's heat equation theorem, it is convenient to introduce the space $\widehat{G}_{n}^{s}$ of class $s$ Gevrey series in $n$ variables (Gevrey 1913, 1918) (see also Sect. 4 below). These are formal power series:

$$
\sum_{I \in \mathbb{N}^{n}} a_{I} z^{I} \in \mathbb{C}[[z]]
$$

such that

$$
\sum_{I \in \mathbb{N}^{n}} a_{I} \frac{z^{I}}{(\sigma(I) !)^{s-1}}
$$

is convergent in a sufficiently small neighbourhood of the origin. For $s=1$, Gevrey series are just analytic series, for $s<1$ these are entire functions and for $s>1$ this class contains divergent power series such as $\sum_{n}(n !)^{s-1} z^{n}$.

Theorem 1.1 (von Kowalevsky 1875) The formal solution to the one dimensional heat equation

$$
\partial_{t} u=\partial_{z z} u, \quad u(t=0,-)=u_{0}, u_{0} \in \widehat{G}_{1}^{s}
$$

1. is a Gevrey class 2 s series;

2. has a unique holomorphic solution if $u_{0} \in \widehat{G}_{1}^{1 / 2}$; 
3. is divergent if $u_{0} \notin \widehat{G}_{1}^{1 / 2}$.

Time evolution for the heat equation theorem became a classical subject and was treated in details in Hadamard's lectures on partial differential equations (Hadamard 1932).

In the 1980s, Ouchi made an important step, when he discovered that the divergent series associated to time evolution of a single linear partial differential equation are always asymptotic expansions of sectorial solutions (Ouchi 1983) (see also Tahara 2011a,b; Yonemura 1990 and references therein). We will extend the results of Kovalevskaïa thesis and Ouchi's theorem to arbitrary systems of linear partial differential equations with Gevrey coefficients.

\section{The Euler Differential Equation}

Before stating our results, we briefly recall basic facts on divergent power series.

Consider the following differential equation of Euler type: ${ }^{1}$

$$
t^{2} \frac{d u}{d t}+(t-1) u+1=0, \quad u(0)=1 .
$$

The formal solution to this differential equation is the divergent series of Gevrey class 2:

$$
u(t)=\sum_{n \geq 0} n ! t^{n} .
$$

We perform a Borel transform:

$$
t^{k} \mapsto \frac{\xi^{k}}{k !}
$$

and get a convergent power series which extends as a meromorphic function in the complex plane:

$$
v(\xi)=\sum_{n \geq 1} \xi^{n}=\frac{1}{1-\xi} .
$$

The functions $v$ and $u$ are related via a Laplace type integral. Indeed, a direct computations shows that:

$$
\frac{1}{t} \int_{0}^{+\infty} e^{-\frac{\xi}{t} \xi^{k}} d \xi=k ! t^{k}
$$

Thus for any polynomial, we get:

\footnotetext{
1 For computational reasons, I slightly modified Euler original example (Euler 1760).
} 


$$
\frac{1}{t} \int_{0}^{+\infty} e^{-\frac{\xi}{t}}\left(\sum_{k=0}^{n} a_{k} \xi^{k}\right) d \xi=\sum_{k=0}^{n} k ! a_{k} t^{k}
$$

More generally, consider a holomorphic power series:

$$
\xi \mapsto v(\xi)=\sum_{n \geq 0} a_{n} \xi^{n}
$$

which extends holomorphically to a neighbourhood of the real positive half-line with at most exponential growth:

$$
\exists r, A>0, \quad|v(\xi)| \leq A e^{\xi / r}
$$

Then the exponential integral:

$$
u(t)=\frac{1}{t} \int_{0}^{+\infty} e^{-\frac{\xi}{t}} v(\xi) d \xi
$$

is holomorphic in the "half-disk":

$$
\{t \in \mathbb{C}: \operatorname{Re} t>0,|t|<r\}
$$

and its asymptotic expansion at the origin is:

$$
\sum_{n \geq 0} n ! a_{n} t^{n}
$$

However, in our example, the function

$$
v(\xi)=\frac{1}{1-\xi}
$$

is not holomorphic in a neighbourhood of the real positive half-line: it has a meromorphic singularity at $\xi=1$. Therefore, in order to define the exponential integral

$$
u(t)=\frac{1}{t} \int_{\gamma} e^{-\frac{\xi}{t}} v(\xi) d \xi
$$

we need to choose a path $\gamma$ which avoids this singularity. This can be done in several ways, for instance we get two different paths $\gamma_{ \pm}$by avoiding the singularity in the upper half-plane or in the lower one (Fig. 1).

Fig. 1 Deformation of $[0,+\infty)$

into $\gamma_{+}, \gamma_{-}$

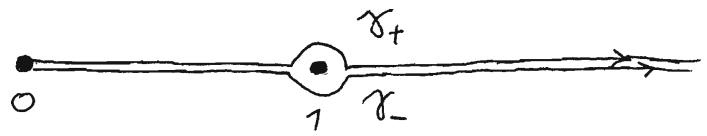


For both paths, the exponential integral is now well-defined:

$$
u_{ \pm}(t)=\frac{1}{t} \int_{\gamma_{ \pm}} e^{-\frac{\xi}{t}} \frac{1}{1-\xi} d \xi
$$

The formal solution

$$
u(t)=\sum_{n \geq 0} n ! t^{n}
$$

is thus the asymptotic expansion of infinitely many functions. This explains why the solution is divergent: if it were convergent then it would be the solution of a unique holomorphic function. It was already known at the beginning of the twentieth century that a similar phenomenon holds for the heat equation.

Still we need to check that $u_{ \pm}$are actual solutions to our initial differential equation and not just formal ones, that is:

$$
t^{2} \frac{d u_{ \pm}}{d t}+(t-1) u_{ \pm}+1=0
$$

The Laplace integral transformation maps the ring $\mathcal{D}_{t}$ of partial differential operator on $t$ to a convolution algebra on $\xi$ according to the rules:

$$
\begin{aligned}
& \mathcal{D}_{t} \rightarrow \widehat{\mathcal{D}}_{\xi} \\
& t \cdot \mapsto \xi \star \\
& \partial_{t} \mapsto \partial_{\xi}+\xi \partial_{\xi}^{2}
\end{aligned}
$$

The Laplace transform of our differential operator:

$$
P=t^{2} \partial_{t}+t-i d+1
$$

gives a new operator:

$$
\widehat{P}=\frac{1}{2} \xi^{2} \star\left(\partial_{\xi}+\xi \partial_{\xi}^{2}\right)+\xi \star-i d+1
$$

which admits $v(\xi)=1 /(1-\xi)$ as holomorphic solution in the neighbourhood of our integration path. Thus

$$
P\left(u_{ \pm}\right)=\frac{1}{t} \int_{\gamma_{ \pm}} e^{-\frac{\xi}{t}} \widehat{P}\left(\frac{1}{1-\xi}\right) d \xi=0 .
$$

The difference between our two solutions can be computed using the residue theorem:

$$
u_{-}-u_{+}=\frac{1}{t} \int_{\alpha} e^{-\frac{\xi}{t}} \frac{1}{1-\xi} d \xi=2 \sqrt{-1} \pi e^{-1 / t}
$$


where $\alpha$ is a small loop around $\xi=1$. We already knew that the difference between our two solutions should be a flat function but this computation shows that it is of exponential type. This is of course not a coincidence as we shall now see.

\section{Gevrey Asymptotics}

In the above example, all data are globally defined but in this paper we shall be concerned with local assumptions. The problem of local resummability is slightly more involved. Let us start with the basic definitions of local asymptotic analysis.

A closed sector of width $\alpha \in] 0,2 \pi$ ] and radius $r$ is a subset of the form:

$$
\Sigma:=\left\{t \in \mathbb{C}:|t| \leq r ;|\arg t-\theta| \leq \frac{\alpha}{2}\right\}
$$

for some direction $\theta \in S^{1}$. Its interior is an open sector or simply a sector. The direction $\theta$ will play no role in this paper, so we fix $\theta=0$ for simplicity.

Given a Gevrey class $\alpha$ power series

$$
\hat{u}(t)=\sum_{n \geq 0} a_{n} t^{n},
$$

we search for an open sector $\Sigma$ and a holomorphic function

$$
u: \Sigma \rightarrow \mathbb{C}
$$

such that

$$
\lim _{t \rightarrow 0} u^{(i)}(t)=i ! a_{i}
$$

where the limit is taken along any segment contained in $\Sigma$. It can be shown that this is equivalent to the condition for any $n$ there exists a constant $C_{n}$ :

$$
\left|u(t)-\sum_{i \leq n} a_{i} t^{i}\right| \leq C_{n}|t|^{n+1}
$$

in any closed sector contained in $\Sigma$. In such case, we say that $\hat{u}$ is the Poincaré asymptotic expansion of $u$. If moreover $C_{n}$ can be chosen of the form

$$
C_{n}=A^{n}(n !)^{\alpha-1},
$$

we say that it is the Gevrey class $\alpha$ asymptotic expansion of $u$. For instance, the "Euler series":

$$
\sum_{n \geq 0}(-1)^{n} n ! t^{n}
$$


is the Gevrey class 2 asymptotic expansion of the Euler type function:

$$
E(t)=\frac{1}{t} \int_{0}^{+\infty} e^{-\xi} t \frac{1}{1+\xi}
$$

Holomorphic functions which admit a Gevrey class $\alpha \in] 1,3]$ asymptotic expansion in a sector of "maximal" width:

$$
\Sigma_{r}:=\left\{t \in \mathbb{C}:|t| \leq r ;|\arg t|<(\alpha-1) \frac{\pi}{2}\right\},
$$

for some radius $r$ form an algebra. We denote it by $G_{1}^{\alpha}$ and more generally by $G_{n}^{\alpha}$ in case of $n$-variables. The definition extends to values of $\alpha>3$, if we consider functions and sectors over the universal covering of a pointed disk (see Malgrange (1995), p. 190 , for details).

For instance, the above function $E(t)$ belongs to $G_{1}^{2}$ : it is a Gevrey class 2 asymptotic expansion in the half-plane $\{\operatorname{Re} t>0\}$.

Proposition 3.1 For any $\alpha>1$, the map which associates to a Gevrey function its asymptotic expansion

$$
G_{1}^{\alpha} \longrightarrow \widehat{G}_{1}^{\alpha}
$$

is surjective: for any class $\alpha$ Gevrey power series $\hat{u}$ there exists a sector

$\Sigma_{r}$ of width $(\alpha-1) \pi$ and a holomorphic function:

$$
u: \Sigma_{r} \rightarrow \mathbb{C}
$$

such that $\hat{u}$ is the Gevery asymptotic expansion of $u$ at the origin.

Sketch of the proof. The Borel transform:

$$
\mathcal{B}: \widehat{G}_{1}^{\alpha} \rightarrow \widehat{G}_{1}^{1}=\mathcal{O}_{1}, \sum_{i \geq 0} a_{i} t^{i} \mapsto \sum_{i \geq 0} \frac{a_{i}}{\Gamma(1+(\alpha-1) i)} \xi^{i}
$$

associates a holomorphic function $v(\xi)$ to the formal power series $\hat{u}$. In general the function $v$ is defined only in a small neighbourhood of the origin. We take $r>0$ smaller than the convergence radius of $v$ and define the partial Laplace transform:

$$
u(t):=\frac{1}{t^{k}} \int_{0}^{r} e^{-\left(\frac{\xi}{t}\right)^{k}} v(\xi) d\left(\xi^{k}\right)
$$

with $k=1 /(\alpha-1)$. A direct computation shows that $\hat{u}$ is the Gevrey asymptotic expansion of $u$. This proves the proposition. 
The following proposition shows that the ambiguity of Gevrey series considered as asymptotic expansions is, in fact, exponentially small:

Proposition 3.2 For $\alpha>1$, the kernel of the map which associates to a Gevrey function its asymptotic expansion

$$
G_{1}^{\alpha} \rightarrow \widehat{G}_{1}^{\alpha}
$$

consists of functions which are exponentially decreasing of exponent $1 /(\alpha-1)$ :

$$
\exists A, B>0,|u(t)| \leq A e^{-B /|t|^{k}}, k:=\frac{1}{\alpha-1}
$$

in closed subsectors.

The content of this section goes back to Borel, Gevrey, Goursat, Holmgren and others (see Hadamard 1932). Resummation methods were already used in quantum mechanics in the twenties and are standard in quantum field theory (see for instance Landau and Lifschitz 1967). Borel summability was extended to arbitrary Gevrey series by (Écalle 1981 and Ramis 1980). Other recent references are Balser's textbook and Malgrange's lectures on divergent power series (Balser 1994; Malgrange 1995).

\section{Main Theorem}

After these preliminaries, we may now state our theorem on time evolution. First, we need the following non-degeneracy condition: we say that a linear partial differential operator of order s is non-degenerate, if after a change of variables, it can be written in the form:

$$
A_{s} \partial_{z_{n}}^{s}-\sum_{\sigma(I)<s} A_{I} \partial^{I}, \quad A_{I} \in M\left(m, G_{n}^{\alpha}\right)
$$

and $A_{s}$ is an invertible matrix. Here $M(m, R)$ stands for $m \times m$ matrices with entries in the ring $R$ for some positive integer $m$. The main result of this paper is the:

Theorem 4.1 Let $\sum_{\sigma(I) \leq s} A_{I} \partial^{I} u$ be a non-degenerate linear operator with $A_{I} \in$ $M\left(m, G_{n}^{\alpha}\right)$ for some $\alpha \leq 1$. The initial value problem

$$
\partial_{t} u=\sum_{\sigma(I) \leq s} A_{I} \partial^{I} u, \quad u_{0}:=u(t=0,-) \in\left(G_{n}^{\alpha}\right)^{m}
$$

admits solutions $u \in G_{1}^{\alpha s} \hat{\otimes} G_{n}^{\alpha}$.

The theorem says that the formal solution is the asymptotic expansion of holomorphic solutions which are of Gevrey class $\alpha s$ in the time variable and of Gevrey class 
$\alpha$ in the others. For instance, when $\alpha=s=1$, the solution is holomorphic. This is the linear version of the classical Cauchy-Kovalevskaïa theorem (in this particular example, our proof gives the non-linear version as well).

If we take a non-degenerate operator of order $s=2$, the theorem says that the solution is of class $2 \alpha$ in the time variable. For instance, if we consider the heat equation

$$
\partial_{t} u=\sum_{i=1}^{n} \partial_{z_{i}}^{2} u=\Delta u
$$

and take an initial data with $\alpha=1 / 2$ then, as Kovalevskaïa proved for $n=1$, the solution is unique and holomorphic. But if we only take a holomorphic initial condition then the solutions will be of Gevrey class 2 and non unique.

Note that by gluing two solutions in opposite sectors we get that any analytic initial data gives a $C^{\infty}$ solution on the real axis.

In the statement of the theorem, we used a topological tensor product which can simply be understood as the space of functions which have asymptotic expansions of Gevrey class $\alpha s$ (resp. $\alpha$ ) in the $t$ variable (resp. $z$ variable). For more details on topological tensor products see Grothendieck (1955).

There are two main ingredients in the proof of Theorem 4.1:

(i) a generalisation of Cauchy's méthode des majorantes to general flows in infinite dimensional spaces,

(ii) a Cauchy-Kovalevskaiia theorem for flat functions.

It would be interesting to extend the above theorem to the non-linear case. Let us now comment the theorem in the most simple examples.

Example 4.2 Consider the initial value problem

$$
\partial_{t} u=\partial_{z} u, \quad u(t=0,-)=\frac{1}{1-z} \in G_{1}^{1}=\mathcal{O}_{1} .
$$

The solution is unique and holomorphic:

$$
u(t, z)=\frac{1}{1-(z+t)} .
$$

Note that the singularity at $z=1$ propagates with time.

Example 4.3 Consider the Kovalevskaïa example:

$$
\partial_{t} u=\partial_{z}^{2} u, \quad u(t=0,-)=\frac{1}{1-z} \in G_{1}^{1}=\mathcal{O}_{1} .
$$

Here $\alpha=1, s=2$ so that $\Sigma$ is of the form

$$
\Sigma=\Sigma_{1} \times D_{1}(r)
$$


where $\Sigma_{1}$ is a sector of width $\pi$, that is, a half plane. The theorem states that there exists a holomorphic function

$$
u(t, z):\left\{(t, z) \in D_{2}(r)^{*}: \operatorname{Re} t>0\right\} \rightarrow \mathbb{C}
$$

which satisfies the heat equation and whose expansion at the origin is the formal solution. This result was already known at the beginning of the twentieth century by Gevrey, Hadamard, Holmgren and others. It is also a particular case of Ouchi's theorem.

Observe that in real analysis, the solution of the heat equation in the circle can be solved by Fourier series and the flow is only defined for positive time. The situation is here completely different, since it admits solutions both for positive and negative time.

The above theorem implies that for a sufficiently regular initial condition, the solution is unique and holomorphic:

Corollary 4.4 There is a unique holomorphic solution to an initial value problem of the form

$$
\partial_{t} u=\sum_{\sigma(I) \leq s} A_{I} \partial^{I} u, \quad u_{0}:=u(t=0,-) \in\left(G_{n}^{1 / s}\right)^{m}, A_{I} \in M\left(m, G_{n}^{1 / s}\right) .
$$

Example 4.5 There is a unique holomorphic solution to the initial value problem.

$$
\partial_{t} u=\partial_{z}^{2} u, \quad u(t=0,-)=e^{z} \in G_{1}^{0} .
$$

This is a particular case of Kovalevskaïa's heat equation theorem.

\section{Formal Evolution}

From a purely heuristic point of view, an evolution equation defines an infinite dimensional dynamical system. This is of course an approximative assertion because these infinite dimensional flows are in general ill-defined. Our first task is to clarify this point.

In the linear case, this is quite obvious. Let $E$ be a topological vector space over a topological field $k$ and

$$
L: E \rightarrow E
$$

a linear map. We denote by $E[[t]]:=k[[t]] \hat{\otimes} E$ the vector space of formal power series with coefficients in $E$ :

$$
\sum_{n \geq 0} t^{n} \otimes a_{n}
$$


that is the projective limit of the vector spaces $k[[t]] /\left(t^{n}\right) \otimes_{k} E$.

The map $L$ induces a map id $\otimes L$ on $E[[t]]$ :

$$
(\mathrm{id} \otimes L)\left(\sum_{n \geq 0} t^{n} \otimes a_{n}\right)=\sum_{n \geq 0} t^{n} \otimes L\left(a_{n}\right) .
$$

We abuse notation and write $L$ for id $\otimes L$. Similarly, we write $\partial_{t}$ instead of $\partial_{t} \otimes \mathrm{Id}$ and so on. We also identify $E$ with the subspace $1 \otimes E \subset E[[t]]$. If the field $k$ is of characteristic zero then the exponential is well-defined and $e^{t L} u_{0}$ is the unique solution to the initial value problem

$$
\partial_{t} u=L u, \quad u(t=0,-)=u_{0} .
$$

The operator $e^{t L}$ is called the time evolution of the operator $L$.

In order to extend this definition of evolution to non-linear operators, we construct the Lie derivative in the infinite dimensional context. First, we recall basic notions on holomorphic maps.

Let $E, F$ be locally convex vector spaces and let $U \subset E$ denote an open subset. A mapping:

$$
f: U \rightarrow F
$$

is called Gâteaux differentiable at a point $u \in U$, if for any $\xi \in E$, the following limits exists

$$
D f(u) \xi:=\lim _{t \mapsto 0} \frac{f(u+t \xi)-f(u)}{t} .
$$

We would like to find some vector space of functions from $E$ to $F$ which is stable under differentiation. In finite dimensional differential geometry, one may choose the space of $C^{\infty}$ functions but differentiability is difficult to handle in the infinite dimensional context (except for the case of Banach spaces). Therefore, we now assume that $k=\mathbb{C}$ and consider the space of holomorphic maps from $E$ to $F$, denoted $\mathcal{H}(E, F)$. These are defined as follows:

Definition 5.1 A mapping $f: E \supset U \rightarrow F$ is called holomorphic if it satisfies the following two conditions:

(i) it is continuous,

(ii) for any continuous linear mappings $j: \mathbb{C} \rightarrow E, \pi: F \rightarrow \mathbb{C}$ the map $\pi \circ f \circ j$ is holomorphic.

For instance, a linear mapping is holomorphic if and only if it is continuous. Like in the finite dimensional case, holomorphic functions in infinitely many variables are given by convergent analytic power series. In particular, if a series is convergent it corresponds to a unique holomorphic map. 
The study of holomorphic functions in infinitely many variables goes back to the beginning of the twentieth century. I have followed Dineen's textbook (Dineen 1981) to which the reader may refer for more details on the subject. Let us now proceed to the definition of formal time evolution in the non-linear case.

We call elements of $\mathcal{H}(U, E)$ holomorphic vector fields in $U$. By contracting the differential with a vector field $X \in \mathcal{H}(U, E)$, we define the Lie derivative

$$
L_{X}: \mathcal{H}(U, E) \rightarrow \mathcal{H}(U, F), \quad f \mapsto[u \mapsto D f(u) X(u)]
$$

for general locally convex spaces. As the Lie derivative is a linear map, this defines the derivation associated to a vector field in the infinite dimensional context.

Now consider the particular case $E=F$. The Lie derivative

$$
L_{X}: \widetilde{E} \rightarrow \widetilde{E}, \quad \widetilde{E}=\mathcal{H}(U, E)
$$

being a linear map, we are back to the situation of linear evolution. Therefore, time evolution of any mapping

$$
f: \widetilde{E} \rightarrow \widetilde{E}
$$

exists. Now the vector space $\widetilde{E}$ contains a distinguished element: the identity.

Definition 5.2 The formal flow of a holomorphic vector field $X \in \mathcal{H}(U, E)$ at $u_{0}$ is the evaluation of the map $e^{t L_{X}}$ Id at $u_{0}$, where $L_{X}$ is the Lie derivative along $X$.

Note that by construction the flow is a solution of the differential equation

$$
\frac{d u}{d t}=\left(L_{X} \operatorname{Id}\right)(u)=X(u)
$$

Thus, we defined evolution for non-linear vector fields in $E$. A convergent formal power series defines in a unique way a holomorphic function, in the infinite dimensional situation as well. Therefore the unicity of formal evolution implies the unicity of holomorphic evolution when it exists.

Example 5.3 Consider the inviscid Burgers equation:

$$
\partial_{t} u=u \partial_{z} u, \quad u(t=0, \cdot)=u_{0} .
$$

Here the vector field is

$$
X: \mathbb{C}\{z\} \rightarrow \mathbb{C}\{z\}, \quad u \mapsto u \partial_{z} u
$$

where $\mathbb{C}\{z\}$ is the vector space of convergent power series in one variable $z$, it has a natural topology (see e.g. Grothendieck 1973). The Lie derivative

$$
L_{X}: \mathcal{H}(\mathbb{C}\{z\}) \rightarrow \mathcal{H}(\mathbb{C}\{z\}), \quad[u \mapsto f(u)] \mapsto[u \mapsto D f(u) u \partial u]
$$


of the identity is.

$$
f=L_{X} \mathrm{Id}: \mathbb{C}\{z\} \rightarrow \mathbb{C}\{z\}, \quad u \mapsto u \partial_{z} u .
$$

The function $f$ is bilinear thus the Gâteaux differential gives

$$
D f(u) \xi=\xi \partial_{z} u+u \partial_{z} \xi .
$$

Substituting $\xi$ by $X$ gives time evolution up to order 2:

$$
\left(e^{t \otimes L} \mathrm{Id}\right) u=u+t u \partial_{z} u+\frac{t^{2}}{2}\left(2 u\left(\partial_{z} u\right)^{2}+u^{2} \partial_{z}^{2} u\right)+o\left(t^{2}\right) .
$$

In simple words, the possibility to define differential calculus in the space of holomorphic functions $\mathcal{H}(\mathbb{C}\{z\})$ allows us to define formal flows like for the finite dimensional spaces. This explains the unicity of the formal and holomorphic solutions to initial value problems.

\section{Generalisation of the Heat Equation Theorem}

Lutz-Myiake-Schäfke proposed to study the heat equation, under global assumptions, via Borel resummation procedure (Lutz 1999). Although we will not deal with the global problem of resummability, it is worthwhile to look back at the Kovalevskaiia example from their point of view.

We start with the heat equation

$$
\partial_{t} u=\partial_{z z} u
$$

with initial value:

$$
u_{0}:(\mathbb{C}, 0) \rightarrow(\mathbb{C}, 0), \quad z \mapsto \frac{1}{1-z}
$$

The formal power series expansion of this Cauchy problem is of Gevrey class 2

$$
\hat{u}(t, z)=\frac{1}{1-z} \sum_{k \geq 0} \frac{(2 k) !}{k !} \frac{t^{k}}{(1-z)^{2 k}} .
$$

The Borel transform of $\hat{u}$ is:

$$
v(\xi, z)=\frac{1}{1-z} \sum_{k \geq 0} \frac{(2 k) !}{(k !)^{2}} \frac{\xi^{k}}{(1-z)^{2 k}}
$$


It extends as an algebraic function

$$
v(\xi, z)=\frac{1}{\sqrt{(1-z)^{2}-4 \xi}} .
$$

The function $v$ can be followed along any path starting from the origin which avoids the curve of equation:

$$
\xi=\frac{(1-z)^{2}}{4}
$$

As before we define paths $\gamma_{ \pm}$avoiding the singularity $\xi=1 / 4$ from above and from below. For $z$ sufficiently close to the origin, the paths also avoids the singularities $\left(1-z^{2}\right) / 4$. We are now in a situation similar to that of the Euler equation: the power series $\hat{u}$ is the asymptotic expansion at $t=0$ of the functions

$$
u_{ \pm}(z, t):=\frac{1}{t} \int_{\gamma_{ \pm}} e^{-\frac{\xi}{t}} \frac{1}{\sqrt{(1-z)^{2}-4 \xi}} d \xi
$$

for $z$ sufficiently small.

As explained above, the ring of partial differential operators $\mathcal{D}_{t, z}$ is mapped to $\widehat{\mathcal{D}}_{\xi, z}$. The function

$$
v(\xi, z)=\frac{1}{\sqrt{(1-z)^{2}-4 \xi}}
$$

is a solution of the partial differential equation:

$$
\left(\partial_{\xi}+\xi \partial_{\xi}^{2}\right) v=\partial_{z}^{2} v
$$

and

$$
\left(\partial_{t}-\partial_{z}^{2}\right) u_{ \pm}=\frac{1}{t} \int_{\gamma_{ \pm}}\left(\partial_{\xi}+\xi \partial_{\xi}^{2}-\partial_{z}^{2}\right) v(\xi) d \xi=0
$$

This means that the functions $u_{ \pm}$are solutions to our initial value problem. Like for the Euler equation, the divergence of the asymptotic series is related to the nonuniqueness of the solution.

Now back to formal solutions.

Theorem 6.1 (Garay 2008) The formal solution to an initial value problem

$$
\partial_{t} u=\sum_{\sigma(I) \leq s} A_{I} \partial^{I} u, \quad u_{0}:=u(t=0,-) \in\left(\widehat{G}_{n}^{\alpha}\right)^{m}
$$


of order $s$ is of Gevrey class $\alpha$ s in the time variable, that is, time evolution defines a map:

$$
e^{t L} \operatorname{Id}:\left(\widehat{G}_{n}^{\alpha}\right)^{m} \rightarrow\left(\widehat{G}_{n}^{\alpha}\right)^{m} \hat{\otimes} \widehat{G}^{\alpha s}, \quad u_{0} \mapsto\left(e^{t L} \mathrm{Id}\right) u_{0}
$$

where $L$ is the Lie derivative associated to the operator.

For a single partial differential equation $(m=1)$ with holomorphic initial data $(\alpha=1)$, the theorem is due to Ouchi. Using techniques due to Boutet de Monvel and Kree, Yonemura simplified the proof (Boutet de Monvel and Krée 1967; Ouchi 1983; Yonemura 1990). Our proof will be self-contained.

Gevrey properties for some particular systems of partial differential equations (other than the heat equation) is proved in Fernández-Fernández and Castro-Jiménez (2011). In the non-linear case, Tahara proved that $C^{\infty}$ solutions, when they exist, are always Gevrey regular (Tahara 2011a, b). For the moment, no further result seems to be known for non-linear evolution equations. Nevertheless, our proof would easily extend to the non-linear case if we were able to prove the Gevrey regularity of the solution of the initial value problem:

$$
\partial_{t} u=\frac{u}{1-u} \partial_{z}^{k} u, \quad u(t=0,-)=u_{0} .
$$

Unfortunately, I was unable to prove or to disprove this fact.

We postpone the proof of the theorem to Sect. 8 and discuss the relation between formal solutions and asymptotic ones. We face here the same problem than in the one variable case: partial Laplace resummation is not a mapping of $\mathcal{D}$-modules. For instance, for any $r<1 / 3$, the asymptotic expansion at $t=0$, inside the half plane $\operatorname{Re} t>0$, of the function:

$$
f(z, t):=\frac{1}{t} \int_{0}^{r} e^{-\frac{\xi}{t}} \frac{1}{\sqrt{(1-z)^{2}-4 \xi}} d \xi
$$

gives the formal solution to the heat equation with initial data $1 /(1-z)$. Nevertheless, this function does not satisfy the heat equation. The function

$$
\left(\partial_{t}-\partial_{z z}\right) f
$$

is a non zero flat function at $t=0$. Thus we need a Cauchy-Kovalevskaïa type theorem for flat functions. Namely, we search for a solution of the form

$$
u=f+v,
$$

where $v$ is flat at $t=0$.

Now, the heat equation gives:

$$
\left(\partial_{t}-\partial_{z z}\right) v+\left(\partial_{t}-\partial_{z z}\right) f=0
$$


which we re-write as

$$
\partial_{z z} v=\partial_{t} v+h, h=\left(\partial_{t}-\partial_{z z}\right) f
$$

Therefore if we can find a flat function $v$ in the $t$-variable which solves this equation then we are done. More generally for the $n$-dimensional heat equation, we are led to an equation of the form:

$$
\partial_{z_{n}}^{2} v=\partial_{t} v-\sum_{i=1}^{n-1} \partial_{z_{i}}^{2} v+h
$$

where $h$ is flat in the $t$-variable.

\section{Cauchy-Kovalevskaïa Theorem for Flat Functions}

Let us start with the now standard abstract Cauchy-Kovalevskaiia theorem. A Banach scale $\left(E_{i},|\cdot|_{i}\right), i \in I$ is a decreasing chain of Banach subspaces of a complex vector space $E$ indexed by a filtred set $I$ (in the sequel an interval or a product of such). We denote by $B_{E}$ the union of their unit balls:

$$
B_{E}=\bigcup_{r}\left\{x \in E_{r}:|x|_{r} \leq 1\right\}
$$

Theorem 7.1 (Baouendi and Goulaouic 1977; Nagumo 1942; Nirenberg 1972; Nishida 1977; Ovsyannikov 1965) Let $\left.\left.\left(E_{r},|\cdot|_{r}\right), r \in\right] 0,1\right]$ be a chain of Banach spaces and

$$
F: B_{E} \rightarrow E
$$

a holomorphic map for which there exists a constant $C>0$ such that

$$
|F(u)-F(v)|_{r} \leq \frac{C}{\sigma}|u-v|_{r+\sigma},
$$

for any $r \in[0,1[$ and any $\sigma \in] 0,1-r]$. There exists a holomorphic function $u$ : $\{|z|<s\} \rightarrow$ E such that:

$$
\frac{d}{d \tau} u=F(u), \quad u(\tau=0, \cdot)=0 .
$$

As the map is holomorphic, the solution is necessarily unique. Let us first apply this theorem to the original Cauchy-Kovalevskaiia situation. We consider the polydisks:

$$
D_{r}=\left\{z \in \mathbb{C}^{n}:\left|z_{i}\right|<r\right\}
$$


and take $E_{r}$ to be the space of holomorphic functions inside $D_{r}$ which are continuous on its boundary:

$$
E_{r}=C^{0}\left(\bar{D}_{r}\right) \cap \mathcal{O}\left(D_{r}\right)
$$

The supremum norm $|\cdot|_{r}$ induces a Banach space structure on $E_{r}$. The Cauchy integral formula implies the Cauchy inequalities:

$$
\left|\partial_{z_{i}} u\right|_{r} \leq \frac{1}{\sigma}|u|_{s+\sigma}, \quad \sigma>0
$$

These Cauchy inequalities imply that there exists a constant $\mathrm{C}$ such that:

$$
|F(u)-F(v)|_{r} \leq \frac{C}{\sigma}|u-v|_{r+\sigma}
$$

for any non-linear partial differential operator $F$ of order 1 . Now given an initial value problem:

$$
\partial_{\tau} u=F(u), \quad u(\tau=0, \cdot)=u_{0}
$$

we define $v=u+u_{0}$ and get a new initial value problem for $v$ such that $v(t=0, \cdot)=0$. Therefore, the condition of the theorem $u(0)=0$ is not restrictive. This shows that the abstract theorem implies the standard one as a particular case. Note also that the non-autonomous case can be reduced to the autonomous case by adding the time as a new variable. This is the Nagumo approach to the Cauchy-Kovalevskaiia theorem.

The interesting point is that Nagumo's proof can be formulated in abstract terms and that the resulting abstract Cauchy-Kovalevskaïa theorem is valid in much more general situations. For instance, consider the rings:

$$
R_{r}=\left\{z \in \mathbb{C}^{n}: r_{1} \leq\left|z_{i}\right| \leq r_{2}\right\}
$$

The abstract Cauchy-Kovalevskaïa theorem tells us that there is a unique holomorphic solution in any of these. However, it does not imply a solution in the pointed disk. Indeed when $r=\left(r_{1}, r_{2}\right)$ go to zero, the bound for the flow is, in general, not uniform in the $r$ variable.

This difficulty can already be seen for the initial value problem:

$$
\partial_{\tau} u=\partial_{z} u, \quad u(\tau=0,-)=e^{-1 / z} .
$$

The solution is:

$$
u(\tau, z)=e^{-1 /(z-\tau)}
$$


at $\tau=0$ there is a singularity at $z=0$. When $\tau$ moves this singularity propagates so there does not exist flow in a subspace of holomorphic functions in the pointed disk:

$$
D^{*}=\{z \in \mathbb{C}: 0<|z|<1\} .
$$

If such a propagation of singularities occurred in our case then we would be lost. Fortunately this does not happen.

Before stating an abstract Cauchy-Kovalevskaïa theorem for flat functions, we give a variant of the Cauchy inequalities in the sectorial case.

Consider the closed sets:

$$
\Sigma_{r}:=\left\{z \in \mathbb{C}: r_{1} \leq|z| \leq r_{2},|\arg z| \leq 2 r_{3}\right\}
$$

with $r_{3}<\pi / 2$. We introduce the $\mathbb{R}_{+}$-action on $\mathbb{R}^{3}$ defined by:

$$
\left(r_{1}, r_{2}, r_{3}\right)+\sigma=\left(r_{1}-\sigma, r_{2}+\sigma, r_{3}+\sigma\right) \text {. }
$$

We get that:

$$
\forall z \in \Sigma_{r}, \quad z+r_{1} D(\sigma) \subset \Sigma_{r+\sigma}
$$

for $\sigma$ small enough. More generally we say that the Banach scale admits an $\mathbb{R}_{+}$-action if there is an action on its indices compatible with the order.

From this property, we deduce the following variant of the Cauchy inequalities:

Lemma 7.2 For any holomorphic function

$$
u: \Sigma_{r} \rightarrow \mathbb{C}
$$

we have

$$
\left|\frac{d}{d z} u\right|_{r} \leq \frac{1}{r_{1} \sigma}|u|_{r+\sigma},
$$

for $\sigma$ small enough.

Proof Take $z \in \Sigma_{r}$ and denote by $\gamma$ the oriented boundary of the disk $z+r_{1} D(\sigma)$. By integration by part in the Cauchy integral formula:

$$
\frac{d}{d z} u(z)=\frac{1}{2 \sqrt{-1} \pi} \int_{\gamma} \frac{u^{\prime}(\xi)}{\xi-z} d \xi
$$

we get that:

$$
\frac{d}{d z} u(z)=\frac{1}{2 \sqrt{-1} \pi} \int_{\gamma} \frac{u(\xi)}{(\xi-z)^{2}} d \xi
$$


We parametrise $\gamma$ by

$$
\theta \mapsto z+r_{1} \sigma e^{2 i \pi \theta}
$$

and obtain the integral formula:

$$
\frac{d}{d z} u(z)=\frac{1}{r_{1} \sigma} \int_{\theta=0}^{\theta=1} u\left(z+r_{1} \sigma e^{2 i \pi \theta}\right) e^{-2 i \pi \theta} d \theta .
$$

This shows that:

$$
\left|u^{\prime}\right|_{r} \leq \frac{1}{r_{1} \sigma}|u|_{r+\sigma}
$$

and concludes the proof of the lemma.

The lemma shows that from an abstract viewpoint the operator $S=\partial_{z}$ in the sectorial case behaves like the singular operator $S=z^{-1} \partial_{z}$ in the pointed disk. Indeed, consider the Banach spaces:

$$
E_{r}=C^{0}\left(R_{r}\right) \cap \mathcal{O}\left(R_{r}\right)
$$

We define an $\mathbb{R}_{+}$action on the indices by putting

$$
\left(r_{1}, r_{2}\right)+\sigma=\left(r_{1}-\sigma, r_{2}+\sigma\right)
$$

In both cases we have:

$$
|S(u)|_{r} \leq \frac{f(r)}{\sigma}|u|_{r+\sigma}
$$

with $f(r)=1 / r_{1}$.

We may now state our Cauchy-Kovalevskaïa theorem for flat functions ${ }^{2}$ :

Theorem 7.3 Let $\left(E_{r}\right), r \in I$ be a Banach scale together with an $\mathbb{R}_{+}$-action and let

$$
f: I \rightarrow \mathbb{R}_{+}, \quad r \mapsto f(r)
$$

be some decreasing function. Consider a linear initial value problem of order $j>1$ :

$$
\frac{d^{j}}{d \tau^{j}} u(\tau)=K(u)+S(u)+h, \quad \frac{d^{i}}{d \tau^{j}} u(\tau)=0, \quad i<j
$$

where $K, S: E \rightarrow E$ are linear and $h \in E$. Assume that there exists constants $A, B, C$ such that for any indices:

\footnotetext{
2 The exponent which we denoted by $k$ in Proposition 3.2 is now called $\alpha$
} 
(i) $|K(u)|_{r} \leq \frac{C}{\sigma^{j}}|u|_{r+\sigma}$,

(ii) $|S(u)|_{r} \leq \frac{C f(r)}{\sigma}|u|_{r+\sigma}$,

(iii) $|h|_{r} \leq B e^{-A f(r)^{\alpha}}, \alpha:=\frac{1}{j-1}$,

then the system has a unique holomorphic solution $\tau \mapsto u(\tau)$ and there exists constants $A^{\prime}, B^{\prime}, \delta$ such that

$$
|u(\tau)|_{r} \leq B^{\prime} e^{-A^{\prime} f(r)^{\alpha}}
$$

for $|\tau| \leq \delta,|r| \leq \delta$.

Note that from a down-to-earth point of view the definition of the partial differential operator consists of two parts: an operator $K$ whose order is at most $j$ and a singular part $S$ of order one.

Proof Up to multiplication of all norms by a constant, we may assume that $C=e^{-1}$. Then by applying $k$-times the estimate on $L$ over the intervals

$$
[r+i \sigma / k, r+(i+1) \sigma / k], \quad i=0, \ldots, s-1,
$$

we get that:

$$
\left|K^{k}(u)\right|_{r} \leq \frac{e^{-k} k^{j k}}{\sigma^{j k}}|u|_{r+\sigma} \leq \frac{(k !)^{j}}{\sigma^{j k}}|u|_{r+\sigma}
$$

and similarly

$$
\left|S^{k}(u)\right|_{r} \leq \frac{k ! f(r)^{k}}{\sigma^{k}}|u|_{r+\sigma}
$$

To solve our initial value problem, we introduce new variables:

$$
v_{1}=u, \quad v_{2}=\frac{d u}{d \tau}, \ldots, v_{j}=\frac{d^{j-1} u}{d \tau^{j-1}}
$$

and endow $E_{r}^{j}$ with the norm:

$$
\left|\left(u_{1}, \ldots, u_{j}\right)\right|_{r}=\max \left(\left|u_{1}\right|_{r}, \ldots,\left|u_{j}\right|_{r}\right) .
$$

We write the initial value problem as a first order system:

$$
\frac{d}{d \tau} v=M v+H, \quad H=(h, 0, \ldots, 0)
$$


where $M$ is a $j \times j$ matrix of the form:

$$
M=\left(\begin{array}{ccccccc}
0 & 1 & 0 & 0 & \ldots & 0 & 0 \\
0 & 0 & 1 & 0 & \ldots & 0 & 0 \\
0 & 0 & 0 & 1 & \ldots & 0 & 0 \\
\ldots & \ldots & \ldots & \ldots & \ldots & \ldots & \ldots \\
\ldots & \ldots & \ldots & \ldots & \ldots & \ldots & \ldots \\
0 & 0 & 0 & 0 & \ldots & 0 & 1 \\
K+S & 0 & 0 & 0 & \ldots & 0 & 0
\end{array}\right)
$$

Now endomorphisms of $E$ act on endomorphisms of $E^{j}$ by diagonal multiplication:

$$
P M:=\operatorname{diag}(P, \ldots, P) M
$$

and a direct computation gives:

$$
M^{j}=(K+S) I .
$$

where $I$ is the identity $k \times k$ matrix. This shows that the formal solution:

$$
v(\tau)=\sum_{k \geq 0} M^{k} H \frac{\tau^{k+1}}{(k+1) !}
$$

can be written as:

$$
v(\tau)=\sum_{k \geq 0}(K+S)^{k} \sum_{i=0}^{j-1} M^{i} H \frac{\tau^{j k+i+1}}{(j k+i+1) !} .
$$

We have an obvious estimate

$$
|v(\tau)|_{r} \leq \sum_{k \geq 0} \frac{\left|\tau^{j k}\right|}{(j k) !}\left|(K+S)^{k} \sum_{i=0}^{j-1} M^{i} H\right|_{r}
$$

and according to the above estimates (1) and (2), we get that:

$$
\left|(K+S)^{k} \sum_{i=0}^{j-1} M^{i} H\right|_{r} \leq \sum_{n+p=k}\left(\begin{array}{l}
k \\
n
\end{array}\right) \frac{(n !)(p !)^{j} f(r)^{n}}{\sigma^{n} \sigma^{j p}}\left|\sum_{i=0}^{j-1} M^{i} H\right|_{r+\sigma}
$$

As

$$
(j k) ! \geq(j n) !(j p) !, \quad k=n+p
$$


we get that:

$$
|v(\tau)|_{r} \leq \sum_{n, p \geq 0}\left(\begin{array}{c}
n+p \\
n
\end{array}\right) \frac{n !\left|\tau^{j n}\right| f(r)^{n}}{(j n) ! \sigma^{n}} \frac{(p !)^{j}\left|\tau^{j p}\right|}{(j p) ! \sigma^{j p}}\left|\sum_{i=0}^{j-1} M^{i} H\right|_{r+\sigma}
$$

and:

$$
\sum_{n, p \geq 0}\left(\begin{array}{c}
n+p \\
n
\end{array}\right) \frac{n !\left|\tau^{j n}\right| f(r)^{n}}{(j n) ! \sigma^{n}} \frac{(p !)^{j}\left|\tau^{j p}\right|}{(j p) ! \sigma^{j p}}=\sum_{n \geq 0} \frac{n ! \tau^{j n} f(r)^{n}}{(j n) ! \sigma^{n}} \sum_{p \geq 0} \frac{(p !)^{j} \tau^{j p}}{(j p) ! \sigma^{j p}}
$$

Thus, we have shown the estimate:

$$
|v(\tau)|_{r} \leq \sum_{n \geq 0} \frac{\tau^{j n} f(r)^{n}}{((j-1) n) ! \sigma^{n}} \sum_{p \geq 0} \frac{\tau^{j p}}{\sigma^{j p}}\left|\sum_{i=0}^{j-1} M^{i} H\right|_{r+\sigma}
$$

We apply the estimate

$$
\sum_{n \geq 0} \frac{x^{n}}{((j-1) n) !} \leq e^{x^{\alpha}}, \quad \alpha:=\frac{1}{j-1}
$$

with

$$
x=\frac{\tau^{j} f(r)}{\sigma}
$$

and use the assumption on $h$ to deduce the estimate:

$$
|v(\tau)|_{r} \leq \frac{j B}{1-\tau / \sigma} e^{-\left(A+\tau^{j \alpha} / \sigma^{\alpha}\right) f(r)^{\alpha}}
$$

for $\tau$ sufficiently small. This proves the theorem.

We may now conclude the proof of our main theorem:

Proof of Theorem 4.1 Theorem 6.1 implies that the formal solution

$$
\hat{f}(t)=\sum_{k \geq 0} a_{k} t^{k}, \quad a_{k} \in G_{n}^{\alpha}
$$

is of Gevrey class $\alpha s$ in the time variable. We apply the partial resummation procedure in the $t$ variable: first we make a Borel transform and then a partial Laplace integration. This defines a holomorphic map

$$
f: \Sigma_{r} \times U \rightarrow \mathbb{C}^{n}
$$


where $U$ is a neighbourhood of the origin in $\mathbb{C}^{n}$ and

$$
\Sigma_{r}=\left\{t \in \mathbb{C}:|t| \leq r ;|\arg t|<\frac{\pi}{2(\alpha-1)}\right\} .
$$

The formal solution $\hat{f}$ is the asymptotic expansion of the function $f$. We substitute $u$ by $v+f$ in our system of partial differential equations. The non-degeneracy condition implies that this new system can be written in the form:

$$
\partial_{z_{n}}^{s} v=K(v)+B(z) \partial_{t} v+h .
$$

where $K$ is a linear partial differential of order $s$ in the $z_{i}, B$ is a matrix with entries in $G_{n}^{\alpha}$ and $h$ is an exponentially decreasing flat mapping. We search for a solution with initial condition

$$
\partial_{z_{n}}^{i} v(0)=0, \quad i=0, \ldots, s-1 .
$$

According to Lemma 7.2, Theorem 7.3 applies. Thus our initial value problem has a unique solution $v$ which decreases like $h$ when $t$ approaches the origin. The holomorphic mapping

$$
u:=f+v
$$

provides a solution to our original initial value problem. This proves the theorem.

\section{Generalisation of Cauchy's Majorant Method}

To conclude our proof, it remains to prove Theorem 6.1. We consider vector fields in the infinite dimensional Gevrey spaces $\left(\widehat{G}_{n}^{s}\right)^{m}$ and extend the classical Cauchy majorant method by comparing series in these different functional spaces. Via formal Borel transform these topological vector spaces are isomorphic to spaces of convergent power series. They are therefore endowed with a standard topology (see Grothendieck 1973 for the definition of the topology).

Recall that a formal power series

$$
u:=\sum_{I \in \mathbb{N}^{n}} a_{I} z^{I} \in \mathbb{C}[[z]], \quad z=\left(z_{1}, \ldots, z_{p}\right)
$$

is majorated by another formal power series

$$
u:=\sum_{I \in \mathbb{N}^{n}} b_{I} z^{I} \in \mathbb{R}_{+}[[z]]
$$

if, for all $I \in \mathbb{N}^{n}$, we have the estimates:

$$
\left|a_{I}\right| \leq b_{I}
$$


In such cases, we use the notation

$$
u \ll v .
$$

In particular, $u \gg 0$ means that $u$ is a formal power series with real non-negative coefficients.

Definition 8.1 Let $X, Y$ be two vector fields in $\left(\widehat{G}_{n}^{s}\right)^{m}$. A vector field $X$ in $\left(\widehat{G}_{n}^{s}\right)^{m}$ majorates another one $Y$ if for any $u, v \in\left(\widehat{G}_{n}^{s}\right)^{m}$

$$
u \gg v \Longrightarrow X(u) \gg Y(v) \text {. }
$$

In particular $X \gg 0$ means that:

$$
u \gg 0 \Longrightarrow X(u) \gg 0 \text {. }
$$

Example 8.2 Let $X$ be a vector field associated to a linear partial differential operator

$$
X:\left(\mathcal{O}_{n}\right)^{m} \rightarrow\left(\mathcal{O}_{n}\right)^{m}, \quad u \mapsto \sum_{\sigma(I) \leq S} A_{I} \partial^{I} u
$$

Then $X \gg 0$ provided that the entries of the matrices $A_{I}$ are analytic series with real non negative coefficients, i.e., $A_{I} \gg 0$.

The following proposition is a direct consequence of the exponential formula for time evolution:

Proposition 8.3 Let $X, Y$ be two vector fields defined in an open subset of $\left(\widehat{G}_{n}^{s}\right)^{m}$.

(i) If $X \ll Y$ then the flow of $X$ at $u_{0} \gg 0$ is majorated by that of $Y$ at the same point,

(ii) If $X \gg 0$ and $v_{0} \gg u_{0}$ then the flow of $X$ at $u_{0}$ is majorated by that of $X$ at $v_{0}$.

We proceed to the proof of Theorem 6.1 and start with a

Proposition 8.4 The following assertions are equivalent

1. the flow of any linear initial value problem of order s in $\left(\widehat{G}_{n}^{\alpha}\right)^{m}$ is of Gevrey class $\alpha s$ in the time variable,

2. the flow of any linear initial value problem of order $s$ in $\widehat{G}_{n}^{\alpha}$ is of Gevrey class $\alpha s$ in the time variable, ${ }^{3}$

3. the flow of any linear initial value problem of order $s$ at $u_{0}=\sum_{n \geq 0}(n !)^{\alpha-1} z^{n} \in$ $\widehat{G}_{1}^{\alpha}$ is of Gevrey class $s$,

4. the flow of $u \mapsto u_{0} \partial_{z}^{s} u$ at the point $u_{0}=\sum_{n \geq 0}(n !)^{\alpha-1} z^{n}$ is of Gevrey class $\alpha s$.

\footnotetext{
3 Note that Ouchi's theorem implies that this is true for $\alpha=1$.
} 
Proof Let us first make a remark. Consider a vector field defined by a linear differential operator:

$$
X:\left(\widehat{G}_{n}^{\alpha}\right)^{m} \rightarrow\left(\widehat{G}_{n}^{\alpha}\right)^{m}, \quad u \mapsto \sum_{\sigma(I) \leq s} A_{I} \partial_{z}^{I} u ; I=\left(i_{1}, \ldots, i_{n}\right)
$$

at a point $u_{0}$.

The map

$$
\text { abs }: \widehat{G}_{n}^{\alpha} \rightarrow \widehat{G}_{n}^{\alpha}, \quad \sum_{I} a_{I} z^{I} \mapsto \sum_{I}\left|a_{I}\right| z^{I}
$$

induces a map on matrices with coefficients in $\widehat{G}_{n}^{\alpha}$ that we denote in the same way.

Replace, in the initial value problem the $A_{I}$ 's by abs $A_{I}$ 's and $u_{0}$ by abs $u_{0}$. By Proposition 8.3, if the solution of this new initial value problem is of Gevrey class $k$ then $X, u_{0}$ has the same property. Therefore it is sufficient to consider the case $X \gg 0$, $u_{0} \in\{u \gg 0\}$.

(2) $\Longrightarrow(1)$.

Let us consider the linear mapping

$$
\psi:\left(\widehat{G}_{n}^{\alpha}\right)^{m} \rightarrow \widehat{G}_{n}^{\alpha}, \quad\left(u_{1}, \ldots, u_{m}\right) \mapsto \sum_{k=1}^{m} u_{k}
$$

Write $A_{I}=\left(A_{I 1}, \ldots, A_{I m}\right) \gg 0$ and put $f_{I}=\sum_{k} A_{I k}$. For any $u \gg 0$, we have

$$
\begin{aligned}
\psi\left(\sum_{\sigma(I) \leq s} A_{I} \partial^{I} u\right) & =\sum_{k=1}^{m} \sum_{\sigma(I) \leq s} A_{I k} \partial_{z}^{I} u_{k} \ll \sum_{\sigma(I) \leq s}\left(\sum_{k=1}^{m} A_{I k}\right) \partial_{z}^{j}\left(\sum_{k=1}^{m} u_{k}\right) \\
& =\sum_{\sigma(I) \leq s} f_{I} \partial^{I} \psi(u) .
\end{aligned}
$$

The exponential formula for time evolution implies that the image under $\psi$ of the flow of $X$ at $u_{0}$ is majorated by the flow of $\sum_{I} f_{I} \partial^{I}$ at $\psi\left(u_{0}\right)$.

(3) $\Longrightarrow$ (2).

Consider the open subset $U=\{x \gg 0\} \subset \widehat{G}_{n}^{\alpha}$. The mapping

$$
R: \mathbb{C} \rightarrow \mathbb{C}^{n}, \quad z \mapsto(z, \ldots, z)
$$

induces a map

$$
R^{*}: \widehat{G}_{n}^{\alpha}[[t]] \supset U[[t]] \rightarrow \widehat{G}_{1}^{\alpha}[[t]]
$$

An element is of Gevrey class $s$ in the $t$ variable provided that it is the case of its image under $R^{*}$. 
The equalities

$$
R^{*} \partial_{z_{i}} z_{j}^{k}=k z^{k-1} \delta_{i j}, \quad \frac{d}{d z} R^{*} z_{j}^{k}=k z^{k-1}
$$

give the estimate

$$
R^{*} \partial_{z_{i}} \ll \frac{d}{d z} R^{*}
$$

Consider a vector field in $\widehat{G}_{n}^{\alpha}$ for the form:

$$
X: u \mapsto \sum_{I} f_{I} \partial_{z}^{I} u, \quad f_{I} \gg 0
$$

As $R^{*} \partial_{z_{i}} \ll \partial_{z} R^{*}$, the flow of the vector field

$$
\widehat{G}_{1}^{\alpha} \rightarrow \widehat{G}_{1}^{\alpha}, \quad x \mapsto \sum_{\sigma(I) \leq s} R^{*} f_{j} \frac{d^{\sigma(I)} u}{d z^{\sigma(I)}}
$$

at $R^{*} u_{0}, u_{0} \gg 0$ majorates the image under $R^{*}$ of the flow of $X$.

Consider the Gevrey series

$$
f(z):=\sum_{n \geq 0}(n !)^{\alpha-1} z^{n} \in \widehat{G}_{1}^{\alpha} .
$$

and take

$$
u_{0}=\sum_{n \geq 0} a_{n} z^{n} \in \widehat{G}_{1}^{\alpha} .
$$

The series

$$
\sum_{n \geq 0} \frac{a_{n}}{(n !)^{\alpha-1}} z^{n}
$$

is analytic. Thus, by Hadamard's lemma, there exists $A, r>0$ such that

$$
\frac{a_{n}}{(n !)^{\alpha-1}} \leq A r^{n}
$$

This means that the series $u_{0}$ is majorated by $A f(r z)$. If $X \gg 0$, the formal flow passing through $u_{0}$ is majorated by the formal flow passing through $A f(r z)$. Up to multiplication of $u$ and $z$ by constants, we may assume that $A=r=1$.

(4) $\Longrightarrow(3)$. 
Consider the flow of a vector field of the form:

$$
X: u \mapsto \sum_{j=0}^{s} a_{j} \frac{d^{j} u}{d z^{j}}
$$

at $f$ defined above.

As

$$
\frac{d^{s}}{d z^{s}} \gg \frac{d^{j}}{d z^{j}}
$$

for any $j<s$, we get that the flow of $X$ at $u_{0}$ is majorated by that of the vector field

$$
u \mapsto b(z) \frac{d^{s} u}{d z^{s}}, \quad b(z):=\sum_{j=0}^{s} a_{j}(z) .
$$

As before there exists constants $A, r>0$ such that

$$
b(z) \ll A f(r z)
$$

and without loss of generality we may assume, as above, that $A=r=1$. This concludes the proof of the proposition.

To conclude the proof of Theorem 6.1, it remains to prove that the flow of the vector field:

$$
X=f(z) \frac{d^{s}}{d z^{s}}, \quad f(z):=\sum_{n \geq 0}(n !)^{\alpha-1} z^{n}
$$

with initial condition

$$
u_{0}(z)=f(z)
$$

is of Gevrey class $\alpha s$.

In the Banach space of continuous function the multiplication map

$$
g \mapsto f g
$$

is bounded. There is an analogous statement for power series:

Lemma 8.5 For any $\alpha$ there exists a constant $C_{\alpha}>0$ with the following property. Let

$$
f(z):=\sum_{n \geq 0}(n !)^{\alpha-1} z^{n}
$$


and

$$
g:=\sum_{n \geq 0}(n !)^{\alpha-1} a_{n} z^{n}
$$

be such that $\left(a_{n}\right)$ is a real positive increasing sequence then:

$$
f g \ll C_{\alpha} \sum_{n \geq 0}(n !)^{\alpha-1} a_{n} z^{n}
$$

Proof Write

$$
f g=\sum_{n \geq 0} c_{n} z^{n}
$$

with

$$
c_{n}:=\sum_{i+j=n}(i !)^{\alpha-1}(j !)^{\alpha-1} a_{j} \leq\left(\sum_{i+j=n}(i !)^{\alpha-1}(j !)^{\alpha-1}\right) a_{n} .
$$

One easily sees that:

$$
\sum_{i+j=n} i ! j ! \leq 3 n !
$$

For $\alpha \geq 1$, as $\left(a_{n}\right)$ is a positive increasing sequence, we get that:

$$
c_{n} \leq 3^{\alpha-1}(n !)^{\alpha-1} a_{n} .
$$

If $\alpha<1$ we have

$$
\sum_{n \geq 0}\left(\sum_{i+j=n}(i !)^{\alpha-1}(j !)^{\alpha-1}\right)=\left(\sum_{i \geq 0}(i !)^{\alpha-1}\right)^{2}<+\infty .
$$

Consequently the sequence

$$
\left(\sum_{i+j=n}(i !)^{\alpha-1}(j !)^{\alpha-1}\right)_{n \in \mathbb{N}}
$$

tends to zero at infinity. This implies that $c_{n}=o\left(a_{n}\right)$ and concludes the proof of the lemma.

We have

$$
\partial_{z}^{s} f=\sum_{n \geq 0} \frac{(n+s) !}{n !}((n+s) !)^{\alpha-1} z^{n}
$$


The above lemma gives a constant $C_{\alpha}$ such that:

$$
\left(f \partial_{z}^{s}\right)^{k} f \ll C_{\alpha}^{k} \sum_{n \geq 0} \frac{(n+k s) !}{n !}((n+k s) !)^{\alpha-1} z^{n} .
$$

Therefore

$$
u(t) \ll \sum_{n, k \geq 0} C_{\alpha}^{k} \frac{(n+k s) !}{n !} \frac{((n+k s) !)^{\alpha-1}}{k !} z^{n} t^{k}
$$

Let us write $a_{n} \equiv b_{n}$ if the series $\left|a_{n} / b_{n}\right|$ is bounded by a geometric series. We have

$$
\frac{(i+j) !}{i ! j !} \leq 2^{i+j}
$$

thus

$$
(i+j) ! \equiv i ! j !
$$

We get that:

$$
\frac{(n+k s) !}{n !} \frac{((n+k s) !)^{\alpha-1}}{k !} \equiv((\alpha s-1) k) !(n !)^{\alpha-1} .
$$

Thus the flow is of Gevrey class $\alpha s$ in the time variable. This concludes the proof of Theorem 6.1.

Acknowledgments I thank Boris Dubrovin for discussions from which this paper originated back in 2007. Thanks also to Duco van Straten for encouragements and suggestions and to the referees for interesting comments and constructive criticisms.

\section{Appendix: On the Divergence of Formal Solutions}

In Łysik (2004), Łysik proved a result similar to Kovalevskaïa divergence result for the Korteweg-de Vries equation, namely that the solution to the initial value problem:

$$
\partial_{t} u=\partial_{z}^{3} u+u \partial_{z} u, \quad u(t=0, \cdot)=\frac{1}{1+z^{2}}
$$

is not holomorphic (see also Domrin and Domrina 2008; Łysik and Michalik 2008). More generally, one may wonder if our Gevrey estimate for time evolution is optimal. This is indeed the case under very general assumptions:

Theorem 9.1 Consider an evolutionary initial value problem of order $s$

$$
\partial_{t} u=g(u, z) \partial_{z_{1}}^{s} u+G\left(u, \partial^{I_{1}} u, \partial^{I_{2}} u, \ldots, \partial^{I_{k}} u, \widehat{\partial_{z_{1}}^{s} u}\right), \quad \sigma\left(I_{\alpha}\right) \leq s
$$


with $u(t=0, \cdot)=u_{0}$. Assume that $g, G \gg 0$ and $u_{0} \gg 0$. If the convergence radius of the formal Borel transform

$$
\mathbb{C} \rightarrow \mathbb{C}^{m}, \quad z_{1} \mapsto B_{\alpha} u\left(z_{1}, 0, \ldots, 0\right)
$$

is finite then the formal solution to this initial value problem is not of Gevrey class $(\alpha s-\varepsilon)$, for any $\varepsilon>0$.

\section{Proof of Theorem 9.1}

The vector field associated to our initial value problem majorates the vector field

$$
X: u \mapsto g(u, z) \partial_{z_{1}}^{s} u
$$

Moreover, the flow of $X$ at $u_{0}$ obviously majorates that of

$$
Y: u \mapsto g\left(u_{0}, z\right) \partial_{z_{1}}^{s} u
$$

at the same point. Finally, let

$$
\alpha z^{I}, \quad \alpha \neq 0, I \in \mathbb{N}^{n}
$$

be a monomial appearing with a non-zero coefficient in the Taylor expansion of $g$. We have

$$
Y \gg \alpha z^{I} \partial_{z_{1}}^{j}
$$

It remains to prove that the flow of

$$
L=z^{I} \partial_{z_{1}}^{S}
$$

at $u_{0}$ is not of Gevrey class $(\alpha s-\varepsilon)$, for any $\varepsilon>0$.

Given formal power series $f, g$, we write

$$
f \succ g
$$

if there are infinitely many coefficients of $f$ which are greater than that of $g$. If $f \succ g$ and $g$ is not of Gevrey class $s$ then $f$ cannot be of Gevrey class $s$.

Write

$$
u_{0}=\left(u_{0,1}, \ldots, u_{0, m}\right)
$$

Define

$$
f(z):=\sum_{n \geq 0}(n !)^{\alpha-1} z_{1}^{n} .
$$


The assumption on $B_{\alpha s}$ implies that for at least one of the components of $u_{0}$, say $u_{0, j}$, there exists $A, r>0$ such that :

$$
u_{0, j} \succ A f\left(r z_{1}\right)
$$

Up to a multiplication of $z_{1}$ and $u_{0}$ by constants, we may assume that $A=r=1$. As $L=z^{I} \partial_{z_{1}}^{s}$, we have the majorant:

$$
L^{k} \gg z^{k I} \partial_{z_{1}}^{k s}, \quad j \in \mathbb{N} \text {. }
$$

Therefore:

$$
L^{k} f \gg z^{k I} \sum_{n \geq 0}\left(\frac{(n+k s) !}{n !}\right)^{\alpha}(n !)^{\alpha-1} z_{1}^{n} \gg z^{k I} \sum_{n \geq 0}(k !)^{s \alpha}(n !)^{\alpha-1} z_{1}^{n} .
$$

\section{Consequently}

$$
e^{t L_{k}} u_{0} \succ \sum_{k \geq 0, n \geq 0} z^{k I}(k !)^{s \alpha-1}(n !)^{\alpha-1} z_{1}^{n} t^{k}
$$

The right hand-side is not of Gevrey class $s \alpha-\varepsilon$ for any $\varepsilon>0$. This proves the theorem.

\section{References}

Audin, M.: Souvenirs sur Sofia Kovalevskaya. Calvage \& Mounet, Paris (2008)

Balser, W.: From Divergent Power Series to Analytic Functions. Lecture Notes in Mathematics, vol. 1582. Springer, Berlin (1994)

Baouendi, M.S., Goulaouic, C.: Remarks on the abstract form of nonlinear Cauchy-Kovalevsky theorems. Commun. Partial Differ. Equ. 2(11), 1151-1162 (1977)

Boutet de Monvel, L., Krée, P.: Pseudo differential operators and Gevrey classes. Annales de l'Institut Fourier 17(1), 295-323 (1967)

Candelpergher, B., Nosmas, J.C., Pham, F.: Approche de la résurgence. Hermann, Paris (1993)

Dineen, S.: Complex Analysis on Locally Convex Spaces. North Holland Mathematical Studies, vol. 57 (1981)

Domrin, A.V., Domrina, A.V.: On the divergence of the Kontsevich-Witten series. Russ. Math. Surv. 109, 773-775 (2008)

Écalle, J.: Les fonctions résurgentes, vol. 1, algèbres de fonctions résurgentes. Pub. Math. Orsay, 247 (1981)

Euler, L.: De seriebus divergentibus. Novi Commentarii academiae scientiarum Petropolitanae 5, 205-237 (1760) [German translation available on http://www.eulerarchive.org/, E247]

Fernández-Fernández, M.C., Castro-Jiménez, F.J.: Gevrey solutions of the irregular hypergeometric system associated with an affine monomial curve. Trans. Am. Math. Soc. 363, 923-948 (2011)

Garay, M.: On the Gevrey convergence of some characteristic Cauchy problems (2008). arXiv:0710.1753v3 (unpublished)

Gevrey, Sur les fonctions indéfiniments dérivables de classe donnée et leur rôle dans la théorie des équations partielles. Comptes rendus à l'Académie des Sciences, tome 157 num 23, 1913, 1121-1124

Gevrey, M.: Sur la nature analytique des solutions des équations aux dérivées partielles. Annales scientifiques de l'École Normale Supérieure 35(3), 129-190 (1918)

Grothendieck, A.: Espaces vectoriels topologiques. Instituto de Matemàtica Pura e Aplicada, Universidade de São Paulo (1954) [English Translation: Topological Vector Spaces. Gordon and Breach (1973)] 
Grothendieck, A.: Produits tensoriels topologiques et espaces nucléaires. Mem. Am. Math. Soc. 16 (1955)

Hadamard, J.: Le problème de Cauchy et les équations aux dérivées partielles hyperboliques. Hermann et Cie, Paris (1932)

Landau, L., Lifschitz, E.: Mécanique quantique, Physique Théorique, vol. III. MIR, Moscow (1967)

Lutz, D.A., Miyake, M., Schäfke, R.: On the Borel summability of divergent solutions of the heat equation. Nagoya Math. J. 154, 1-29 (1999)

Łysik, G.: Non-analyticity in time of solutions to the KdV equation. Z. Anal. Anwendungen 23(1), 67-93 (2004)

Łysik, G., Michalik, S.: Formal solutions of semilinear heat equations. J. Math. Anal. Appl. 341, 372-385 (2008)

Malgrange, B.: Sommation des séries divergentes. Expositiones Mathematicae 13(2/3), 163-222 (1995)

Nagumo, M.: Über das Anfangswertproblem partieller Differentialgleichungen. Jpn. J. Math. 18, 41-47 (1942)

Nirenberg, L.: An abstract form of the nonlinear Cauchy-Kowalewski theorem. J. Differ. Geom. 6, 561-576 (1972)

Nishida, T.: A note on a theorem of Nirenberg. J. Differ. Geom. 12(4), 629-633 (1977)

Ouchi, S.: Characteristic Cauchy problems and solutions of formal power series. Ann. Inst. Fourier 33, 131-176 (1983)

Ovsyannikov, I.V.: A singular operator in a scale of Banach spaces. Sov. Math. Dokl. 6, 1025-1028 (1965)

Ramis, J.P.: Les series $\kappa$-sommables et leurs applications. In: Iagolnitzer, D. (ed.) Complex Analysis, Microlocal Calculus and Relativistic Quantum Theory, pp. 178-199. Springer, Berlin (1980)

Tahara, H.: Gevrey regularity in time of solutions to nonlinear partial differential equations. J. Math. Sci. Univ. Tokyo 18, 67-137 (2011)

Tahara, H.: Maillet type theorem and Gevrey Regularity in Time of Solutions to Nonlinear Partial Differential Equations. Formal and Analytic Solutions of Differential and Difference Equations, vol. 97. Banach Center Publications, Banach center pp. 125-149 (2011)

von Kowalevsky, S.: Zur Theorie der partiellen Differentialgleichungen. Journal für reine und angewandte Mathematik 80, 1-32 (1875)

Yonemura, A.: Newton polygons and formal Gevrey classes. Publ. RIMS 26, 197-204 (1990)

Zinn-Justin, J.: Quantum Field Theory and Critical Phenomena. Oxford Science Publications, Oxford (1993) 